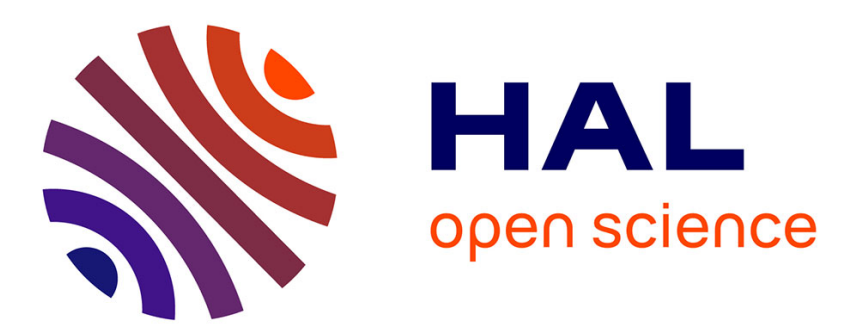

\title{
Combined distributed parameters and source estimation in tokamak plasma heat transport
}

\author{
Sarah Mechhoud, Emmanuel Witrant, Luc Dugard, Didier Moreau
}

\section{To cite this version:}

Sarah Mechhoud, Emmanuel Witrant, Luc Dugard, Didier Moreau. Combined distributed parameters and source estimation in tokamak plasma heat transport. ECC 2013 - 12th biannual European Control Conference, Jul 2013, Zurich, Switzerland. pp.0147. hal-00931650

\section{HAL Id: hal-00931650 \\ https://hal.science/hal-00931650}

Submitted on 15 Jan 2014

HAL is a multi-disciplinary open access archive for the deposit and dissemination of scientific research documents, whether they are published or not. The documents may come from teaching and research institutions in France or abroad, or from public or private research centers.
L'archive ouverte pluridisciplinaire HAL, est destinée au dépôt et à la diffusion de documents scientifiques de niveau recherche, publiés ou non, émanant des établissements d'enseignement et de recherche français ou étrangers, des laboratoires publics ou privés. 


\title{
Combined distributed parameters and source estimation in tokamak plasma heat transport
}

\author{
Sarah Mechhoud ${ }^{1}$, Emmanuel Witrant $^{1}$, Luc Dugard ${ }^{1}$ and Didier Moreau ${ }^{2}$
}

\begin{abstract}
We investigate the joint estimation of time and space distributed parameters and input in the tokamak heat transport equation. This physical phenomenon can be modelled by a non-homogeneous linear parabolic partial differential equation (PDE). The analysis of this PDE is achieved in a finite dimensional framework using the cubic b-splines finite element method. The application of the parameter projection method results in a linear time-varying state-space model with unknown parameters and inputs. The DAISYS method proves the structural identifiability of the model and the EKF-UI-WDF estimates simultaneously the states, parameters and inputs. This methodology is applied on the tokamak plasma heat transport equation in order to reconstruct simultaneously its coefficients and its source term. Computer simulations on both mock-up and real data show the performance of the proposed technique.
\end{abstract}

\section{INTRODUCTION}

Heat transport in tokamak plasmas is one of the main complex physical phenomena in the controlled fusion research. This transport can be described by a one dimensional linear non-homogeneous parabolic partial differential equation with time-varying distributed diffusion coefficient and source term. The reaction parameter is constant. In the thermonuclear fusion community there is no consensus on defined models for these coefficients. For example, there are different empirical and theoretical laws for the diffusion coefficient [1]. Each one depends on various conditions (tokamak dimensions, discharge parameters, temperature profile,...). The heating energy absorbed by the particles (the source term in the heat equation) generally comes from radiofrequency heating or neutral beam injection and is sometimes difficult to model from first principles because of parasitic phenomena and energy losses. Thus, in order to keep the model linear and because of the modelling difficulties, the diffusivity and the source term are assumed to be unknown. The aim of this work is to develop a method to reconstruct, efficiently and simultaneously, these unknown parameters (diffusivity and source term) in the finite dimensional framework. It is important to note that these coefficients are not only useful for the heat transport description but also for the development of current and pressure profile control strategies, which are strongly affected by the temperature

\footnotetext{
${ }^{1}$ S. Mechhoud, E. Witrant and L. Dugard are with UJFGrenoble 1/CNRS, Grenoble Image Parole Signal Automatique (GIPSA-lab), UMR 5216, B.P. 46, F-38402 St Martin d'Hères, France \{sarah.mechhoud, emmanuel.witrant, luc.dugard @gipsa-lab.grenoble-inp.fr

${ }^{2}$ D. Moreau is with CEA, IRFM, F-13108 Saint Paul-Lez-Durance, France didier.moreau@cea.fr
}

dynamics. In this work, our estimation method is an extension of the one used in [2], where the diffusion, reaction and advection parameters of a one dimensional parabolic PDE were estimated, based on a perfect knowledge of the inputs and the measurements. The PDE is discretized using a finite element method based on cubic b-splines [3]. Here the problem considered is to estimate simultaneously the transport parameters and the inputs. The main difference with previous results is the parameter projection. In [2] the parameter projection was performed in time and space, leading to a non-linear optimization problem. Here, a linear estimation problem is derived by projecting the parameters only in space. The resulting time-varying coefficients are then estimated/identified, using the Extended Kalman Filter with Unknown Inputs Without Direct Feedthrough (EKF-UIWDF) [4]. The validation of the estimation strategy relies on our capability to reconstruct the parameters and the inputs, based on the available measurements. This question is answered by analysing the structural global identifiability with the Differential Algebra for Identifiability of SYStems (DAISYS) [5] method. This paper is organized as follows. First, the heat transport model and its cubic b-splines finite element method space-discretization are presented in Section I. The statement of the estimation/identification problem, the structural identifiability and the application of the DAISYS method are in Section II. The joint estimation of the diffusivity and the source term using the EKF-UI-WDF is considered in Section III. Simulations on both illustrative and real-data are carried out to evaluate the performance of the chosen approach.

\section{ELECTRON HEAT TRANSPORT MODEL}

Assuming the poloidal and toroidal axisymmetry, the tokamak is considered as an infinite cylinder where space variations only occur along the radius $r \in[0, a]$. Applying the energy conservation principle and neglecting the spatiotemporel variations of the electron density, the electron heat transport model is given by the following parabolic partial differential equation (PDE) [6]:

$$
\left\{\begin{array}{l}
\frac{\partial T}{\partial t}=\frac{1}{z} \frac{\partial}{\partial z}\left(z \chi_{e}(z, t) \frac{\partial T}{\partial z}\right)-\frac{1}{\tau} T(z, t)+S(z, t) \\
\frac{\partial T}{\partial z}(z=0, t)=0 ; T(z=1, t)=0 \\
\left.T(z, t=0)=0 ; \quad z \in \Omega ; \quad t \in] 0, t_{f}\right]
\end{array}\right.
$$


where $\Omega$ is the interval $] 0,1[, t$ is the time, $a$ the small plasma radius, $\chi_{e}$ the electron diffusivity, $\tau(<\infty)$ a damping time modelling the energy losses, $T$ the electron temperature and $S$ is the power density absorbed by the particles. In system (1), the second and third equations represent initial and mixed Neumann-Dirichlet boundary conditions, chosen to guaranty the symmetry and boundedness of the solution near zero.

The existence and uniqueness conditions of the weak solution $T(z, t) \in L^{2}\left(0, t_{f} ; H_{0,\{1\}}^{1}(\Omega)\right)^{1}$ based on the Lax-Milgramm theorem [7] are satisfied for:

$$
\left\{\begin{array}{l}
\chi_{e} \in L^{2}\left(0, t_{f} ; L^{2}(\Omega)\right) \cap C^{0}\left(0, t_{f} ; C^{1}(\Omega)\right) \\
\text { and } \chi_{e}(x, t) \geq c>0 \\
\tau \in \mathbb{R}_{+}^{*} \\
S \in L^{2}\left(0, t_{f} ; L^{2}(\Omega)\right) \cap C^{0}\left(0, t_{f} ; C^{0}(\Omega)\right)
\end{array}\right.
$$

Once the well-posedness of the heat model is established, an approximate solution can be derived. In this paper, the approximation is considered in the Galerkin formulation, using the cubic b-splines finite element method, one of the widely used methods that provides accurate approximate solutions of class $C^{2}$. In the following, this technique is briefly summarized (see details in [3], [8]).

Starting from the variational formulation of (1), the cubic b-splines basis functions are chosen to span the finite dimensional space of approximate solutions. Specifically, let $\left\{z_{i}\right\}_{i=0}^{n}$ be a uniform mesh of $\Omega$. The approximate solution is given by:

$$
T_{h}(z, t)=\sum_{k=-1}^{n+1} x_{k}(t) \pi_{k}(z)
$$

where $\left\{\pi_{i}\right\}_{i=-1}^{n+1}$ is the sequence of standard piecewise cubic b-splines functions that vanish outside $\left[z_{i-2}, z_{i+2}\right]$ and $\left\{x_{k}(t)\right\}_{n+1}^{k=-1}$ is the sequence of the corresponding weighting functions. However, to satisfy the boundary conditions, some of these functions have to be adapted. Consequently, the modified cubic b-splines basis elements $\left\{\omega_{i}(z)\right\}_{i=0}^{n}$ have the following expression [8]:

$$
\omega_{i}(z)=\left\{\begin{array}{l}
\pi_{0}(z) \quad \text { if } i=0 \\
\pi_{-1}(z)+\pi_{1}(x) \text { if } i=1 \\
\pi_{i}(z), \text { for } i=2, \ldots, n-2 \\
\pi_{n-1}(z)-\pi_{n+1} \text { if } i=n-1 \\
\pi_{n}(z)-4 \pi_{n+1}(z) \text { if } i=n
\end{array}\right.
$$

and the approximate solution (3) becomes:

$$
T_{h}(z, t)=\sum_{k=0}^{n} x_{k}(t) \omega_{k}(z)
$$

Following all the classical steps of a standard b-splines cubic finite element method, the PDE given in (1) is converted to a set of ordinary differential equations (ODE) in the continuous time domain, leading to:

$$
\left\{\begin{array}{l}
\mathfrak{M} \dot{X}(t)=\left(A(t)-\frac{1}{\tau} \mathfrak{M}\right) X(t)+B(t) \\
X(0)=0 .
\end{array}\right.
$$

\footnotetext{
${ }^{1}$ where $L^{2}$ and $H_{0,\{1\}}^{1}$ are two Hilbert spaces [7]
}

where: $X(t)=\left[x_{0}(t), x_{2}(t), \ldots, x_{n}(t)\right]^{T}\left(X \in \mathbb{R}^{n+1}\right)$, $\mathfrak{M} \in \mathbb{R}^{n+1 \times n+1}$ is the stiffness matrix, non-singular (by definition), symmetric and diagonally dominant, written as:

$\mathfrak{M}=\left(\begin{array}{ccc}\int_{0}^{1} \omega_{0}^{2}(z) d z & \ldots & \int_{0}^{1} \omega_{0}(z) \omega_{n}(z) d z \\ \vdots & \ddots & \vdots \\ \int_{0}^{1} \omega_{0}(z) \omega_{n}(z) d z & \ldots & \int_{0}^{1} \omega_{n}^{2}(z) d z\end{array}\right)$

with : $\int_{0}^{1} \omega_{i}(z) \omega_{i+4}(z) d z=0, \quad i=0, \ldots, n-4$.

$A(t) \in \mathbb{R}^{(n+1) \times(n+1)}$ is the matrix of dynamics (or the damping matrix), symmetric and given by:

$$
-\left(\begin{array}{ccc}
\int_{0}^{1} \chi_{e}(z, t) \omega_{0}^{\prime 2}(z) d z & \ldots & \int_{0}^{1} \chi_{e}(z, t) \omega_{0}^{\prime}(z) \omega_{n}^{\prime}(z) d z \\
\vdots & \ddots & \vdots \\
\int_{0}^{1} \chi_{e}(z, t) \omega_{0}^{\prime}(z) \omega_{n}^{\prime}(z) d z & \ldots & \int_{0}^{1} \chi_{e}(z, t) \omega_{n}^{\prime 2}(z) d z
\end{array}\right)
$$

and the input vector $B(t) \in \mathbb{R}^{n+1}$ is given by:

$B(t)=\left(\begin{array}{c}\int_{0}^{1} S(z, t) \omega_{0}(z) d z \\ \vdots \\ \int_{0}^{1} S(z, t) \omega_{n}(z) d z\end{array}\right)$

Splines are a powerful tool for general approximation problems and when combined with finite element methods they provide very accurate approximate solutions. To illustrate this, we compute the Absolute Approximation Error (AAE) defined as:

$$
\left\{\begin{array}{l}
A A E(z, t)=T_{e x p}(z, t)-T_{r e c}(z, t), \quad z \in[0,1] \\
T_{r e c}(z, t)=\sum_{i=0}^{n} \alpha_{i}(t) \omega_{i}(z)
\end{array}\right.
$$

where $T_{r e c}$ is the temperature profile reconstructed after projection of the measured temperature $T_{\exp }$ on the space spanned by $\{\omega\}_{i=0}^{n}$ and $\alpha_{k}$ are the solution of the following Cramer linear system, resulting from the interpolation problem:

$$
\left\{\begin{array}{l}
T_{r e c}\left(z_{i}, t\right)=T_{\exp }\left(z_{i}, t\right), \quad i=0, \ldots, n-1 \\
\frac{\partial T_{r e c}}{\partial z}\left(z_{n}, t\right)=\frac{\partial T_{\text {exp }}}{\partial z}\left(z_{n}, t\right)
\end{array}\right.
$$

As the electron temperature model (1) does not include the edge pedestal and plasma scrape-off phenomena, the AAE is defined for $z$ belonging to the interval $[0,0.8]$. Fig. 1 illustrates the AAE using the Tore Supra experimental data TS 33632 with $n=20$. The AAE can be assimilated to round-off errors.

\section{JOINT DIFFUSION AND SOURCE ESTIMATION}

In this section, we first start with the conversion of the parameter-input $\left(\chi_{e}\right.$ and $S$ ) estimation problem from the infinite to the finite dimensional framework. The fundamental infinite identification problem consists in finding $\chi_{e}$ and $S$ such that: $\chi_{e}>c>0 \in L^{2}\left(0, t_{f} ; L^{2}(\Omega)\right)$ and $S \in$ 


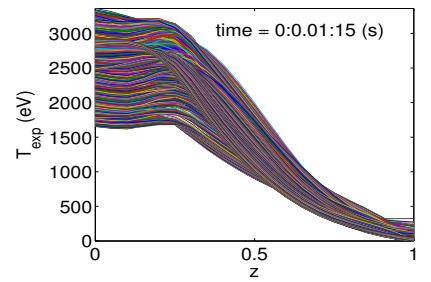

(a)

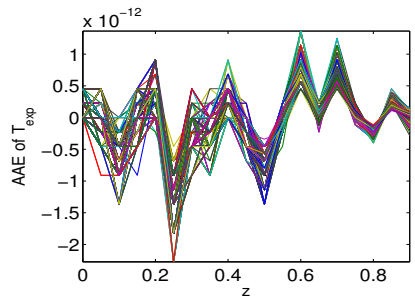

(b)
Fig. 1: (a) Spatio-temporal temperature profile $T_{\exp }$ and (b) its AAE for shot TS 33632.

$L^{2}\left(0, t_{f} ; L^{2}(\Omega)\right)$ which minimize the following distributed least-squares criterion:

$$
J\left(\chi_{e}, S\right)=\sum_{i=0}^{r} \int_{0}^{1}\left(T_{e x p}\left(z, t_{i}\right)-T\left(z, t_{i} ; \chi_{e} ; S\right)\right)^{2} d z
$$

satisfying the constraints (1) and where $T_{\exp }(z, t)$ is the experimental temperature profile given at $t_{i}, i=1, \ldots, r$ and $T\left(z, t ; \chi_{e} ; S\right)$ is the solution of (1).

In order to develop a computationally more tractable estimation solution, (8) is weakened to the following form:

$$
J\left(\chi_{e}, S\right)=\sum_{i=0}^{r} \int_{0}^{1}\left(T_{e x p}\left(z, t_{i}\right)-T_{h}\left(z, t_{i} ; \chi_{e} ; S\right)\right)^{2} d z
$$

where $T_{h}$ is the weak solution of the abstract evolution equation of (1):

$\left\{\begin{array}{l}\frac{d}{d t}\left(T_{h}, v\right)-b\left(T_{h}, v ; t\right)=L(t)(v) ; \forall v \in H_{0,\{1\}}^{1}(\Omega) \\ T_{h}(0)=T(x, 0)=0\end{array}\right.$

where $b$ and $L$ are the bilinear and linear forms associated with (10), uniformly continuous and coercive. This is straightforward considering the spaces defined for $\chi_{e}$ and $S$. Note that the problem (9)-(10) admits a solution if the mapping: $\chi_{e} \longmapsto T_{h}$ is uniformly continuous and $S \longmapsto$ $T_{h}$ is continuous. For more details, refer to [9] and [2], from which the extension to our parameter-input estimation problem is direct (this extension relies on the chosen spaces of $\chi_{e}$ and $S$, which guarantee the existence and uniqueness of equation (10)).

According to the Ritz-Galerkin framework, the approximate solution $T_{h}$ is the orthogonal projection of $T$ on the finite dimensional space (spanned by $\left\{\omega_{i}\right\}$ for example). It converges in $L_{2}$ norm to $T$ as $n \longrightarrow \infty$. Taking $Q_{M}$ (for $\chi_{e}$ ) and $F_{L}$ (for $S$ ) as two compact subsets in $L^{2}\left(0, t_{f} ; L^{2}(\Omega)\right.$ ), the abstract infinite dimensional estimation problem is converted to the following finite one [2]:

$\min _{\chi_{e_{M}}, S_{L}} J^{n}\left(\chi_{e_{M}}, S_{L}\right)=\sum_{i=0}^{r} \sum_{k=0}^{n}\left(\alpha_{k}\left(t_{i}\right)-x_{k}\left(t_{i} ; \chi_{e_{M}} ; S_{L}\right)\right)^{2}$

with the dynamic constraints provided by (6). $\alpha_{k}$ and $x_{k}$ are defined previously in section II, $\left\{\chi_{e_{M}}\right\}$ and $\left\{S_{L}\right\}$ are parameters sequences that converge to the parameter solution $\left(\chi_{e}, S\right)$ under the compactness condition on the chosen parameter sets $\left(Q_{M}\right.$ and $\left.F_{L}\right)$.

For the parameter sets, the space spanned by the cubic piecewise twice differentiable b-splines $\left\{\pi_{k}\right\}_{k=1}^{M, L}$ is chosen. $\chi_{e_{M}}(z, t)$ can be expressed as follows:

$$
\chi_{e_{M}}(z, t)=\sum_{k=1}^{M} \lambda_{k}(t) \pi_{k}(z)=\theta^{T}(t) P(z) .
$$

where:

$$
\left\{\begin{array}{l}
\theta^{T}(t)=\left[\lambda_{1}(t), \lambda_{2}(t), \ldots, \lambda_{M}(t)\right] \quad, \quad \theta \in \mathbb{R}^{M} \\
P(z)=\left[\pi_{1}(z), \pi_{2}(z), \ldots, \pi_{M}(z)\right]^{T}
\end{array}\right.
$$

The source term writes as:

$$
S_{L}(z, t)=\sum_{k=1}^{L} \zeta_{k}(t) \pi_{k}(z)=\mathcal{B}(z) \beta(t) .
$$

where :

$$
\left\{\begin{array}{l}
\beta(t)=\left[\zeta_{1}(t), \zeta_{2}(t), \ldots, \zeta_{L}(t)\right]^{T} \quad, \quad \beta \in \mathbb{R}^{L} \\
\mathcal{B}(z)=\left[\pi_{1}(z), \pi_{2}(z), \ldots, \pi_{L}(z)\right]
\end{array}\right.
$$

The matrix $A(t)$ defined in Section II then becomes:

$$
A\left(\theta^{T}(t)\right)=-\theta^{T}(t) \otimes\left(\begin{array}{c}
\int_{0}^{1} P(z) \omega_{0}^{\prime 2}(z) d z \cdots \int_{0}^{1} P(z) \omega_{0}^{\prime}(z) \omega_{n}^{\prime}(z) d z \\
\ddots \ddots \vdots \\
\int_{0}^{1} P(z) \omega_{0}^{\prime}(z) \omega_{n}^{\prime}(z) d z \cdots \int_{0}^{1} P(z) \omega_{n}^{\prime 2}(z) d z
\end{array}\right)
$$

Since $\mathfrak{M}$ is invertible, (6) can be written as a linear parameter-varying system :

$$
\left\{\begin{array}{l}
\dot{X}(t)=\left(\mathfrak{M}^{-1} A\left(\theta^{T}(t)\right)+\frac{1}{\tau} I_{n+1}\right) X(t)+\mathfrak{M}^{-1} \mathcal{D} \beta(t) \\
X(0)=0 \\
y(t)=X(t)
\end{array}\right.
$$

where $\mathcal{D} \in \mathbb{R}^{(n+1) \times L}$ has the following form:

$$
\mathcal{D}=\left(\begin{array}{ccc}
\int_{0}^{1} \omega_{1}(z) \pi_{1}(z) d z & \cdots & \int_{0}^{1} \omega_{1}(z) \pi_{L}(z) d z \\
\vdots & \ddots & \vdots \\
\int_{0}^{1} \omega_{n}(z) \pi_{1}(z) d z & \cdots & \int_{0}^{1} \omega_{n}(z) \pi_{L}(z) d z
\end{array}\right)
$$

This approach is different from that presented in [2], where the authors were interested only in parameters estimation and where the projection operation was done simultaneously in time and space. This led to a purely nonlinear parameter estimation problem. Note also that, in previous works [10], [11] where the problem was tackled in the infinite dimensional framework, the source term was considered as a known variable and the estimation was computed using nonlinear optimization algorithms performing well for small scaled systems.

The main contribution of our formulation is first to carry out the projection only in space, which allows to finally deal with a linear state-space system with time-varying coefficients. Second, the problem of interest is not to estimate only the parameters but also to reconstruct efficiently and 
simultaneously the inputs.

Once the transformation of the initial problem to the finite dimensional framework is achieved, process identification techniques developed for state-space systems can be used to estimate both $\theta$ and $\beta$ in system (13). But, prior to the estimation process, the question of whether it is possible to estimate the parameters and the input from the available measurements has to be answered. This question is the heart of identifiability.

\section{A. Structural identifiability}

Structural identifiability is related to the model structure, precisely to the injectivity of the input-output mapping $y=\phi(q, u)$, where $q$ is the set of parameters and $u$ the inputs. It is an a priori investigation which attempts to answer the crucial question of whether solutions for the unknown variables of the noise-free model exist or not. The uniqueness of solutions corresponds to the global structural identifiability, the existence of a finite number of solutions to local identifiability and the absence or infinite solutions to non-identifiability.

The local and global structural identifiability of both linear and nonlinear systems with respect to the parameters (not including the inputs) has been exhaustively studied [12], [13] and references therein. One of them, that can be easily understood and extrapolated to unknown inputs identifiability is the Differential Algebra for Identifiability of SYStems method (DAISYS) for which a succinct and clear description can be found in [14].

The application of the DAISYS steps on model (13) is achieved as follows. First the input-output state-independent mapping is computed (our input is noted $\beta$ in (13)):

$\Psi(y, \dot{y}, \beta, \theta, \tau)=\dot{y}+\left(\mathfrak{M}^{-1} P W y\right) \theta+\frac{1}{\tau} y+\mathfrak{M}^{-1} D \beta=0$.

From the $A(\theta(t))$ matrix formula given previously, it is clear that $A$ is linear in $\theta$ and can be readily rearranged so that the first term in the right-side of (13) can be written as:

$$
\left(\mathfrak{M}^{-1} A\left(\theta^{T}(t)\right)+\frac{1}{\tau} I_{n+1}\right) X(t)=\left(\mathfrak{M}^{-1} P W X\right) \theta(t)+\frac{1}{\tau} X
$$

where $P W$ is such that the matrix multiplication is well defined $(\operatorname{dim}(P W)=(n+1) \times M)$.

Second, the injectivity condition is obtained by setting:

$$
\Psi(y, \dot{y}, \beta, \theta, \tau)=\Psi(y, \dot{y}, \stackrel{*}{\beta}, \stackrel{*}{\theta}, \stackrel{*}{\tau})
$$

where $\stackrel{*}{\beta}, \stackrel{*}{\theta}$ and ${ }^{*}$ may be another solution of (14).

(15) can be written as:

$$
\underbrace{\left[\begin{array}{lll}
\mathfrak{M}^{-1} P W y & y & \mathfrak{M}^{-1} D
\end{array}\right]}_{\varphi}\left[\begin{array}{c}
\theta-* \\
\tau-* \\
\tau \\
\beta-\beta
\end{array}\right]=0
$$

Note that the rank of $\varphi$ is equal to $M+1+L \leq n+1$. If $\varphi$ is full column rank (the columns are linearly independent) then $\theta=\stackrel{*}{\theta}, \tau=\stackrel{*}{\tau}$, and $\beta=\stackrel{*}{\beta}$. This means that the parameters $(\theta$ and $\tau$ ) and the inputs $\beta$ are structurally globally identifiable. However, it is not sufficient for the solution's numerical computations: matrix $\varphi$ also has to be well conditioned.

\section{B. Estimation using the EKF-UI-WDF}

The Kalman Filter for Unknown Inputs Without Direct Feedthrough (KF-UI-WDF) is an optimal filter for state and input estimation. Unlike the minimum-variance unbiased (MVU) estimators ([15], [16]) where only the MVU optimality is provided, the KF-UI-WDF is a natural extension of the Kalman filter (KF) to the unknown inputs estimation problem and keeps all the KF performances and practical knowledge. By minimizing a weighted least squares objective function with respect to an extended variable including states and unknown inputs, the filter proposed by [4] guarantees, under classical observability condition, the global optimality for both state and unknown inputs estimation in a least square sense.

In this section the KF-UI-WDF is extended to estimate also the parameter $\theta$ using the same philosophy as the EKF. To this end, we first extend the state vector in (13) to include the unknown parameter $\theta$ and then discretize the time-variation. Define:

$f(X, \theta, \beta, k)=$

$$
\left(\begin{array}{c}
\left(I+d t *\left(\mathfrak{M}^{-1} A\left(\theta^{T}(k)\right)\right)-\frac{1}{\tau} I_{n+1}\right) X(k)+d t * \mathfrak{M}^{-1} \mathcal{D} \beta(k) \\
\theta(k)
\end{array}\right)
$$

The discrete extended model is given by:

$$
\left\{\begin{array}{l}
x_{e x t}(k+1)=f(X, \theta, \beta, k)+w(k) \\
y(k)=C x_{e x t}(k)+v(k)
\end{array}\right.
$$

where $x_{\text {ext }}(k+1)=\left(\begin{array}{c}X(k+1) \\ \theta(k+1)\end{array}\right)$ is the extended state, $C=\left[\begin{array}{ll}I_{n+1} & 0\end{array}\right]$ is the observation matrix, $d t$ is the time step, $w(k) \in \mathbb{R}^{n+1+M}$ and $v(k) \in \mathbb{R}^{n+1}$ are respectively the model uncertainty and the measurements noise vectors, assumed to be independent, white and Gaussian. In order to take account the unknown dynamics of the parameter $\theta$, the model noise $w$ has a dimension of $n+1+M$.

Based on the above representation (16), the EKF-UIWDF approach can be used to estimate the extended state $\hat{x}_{\text {ext }}(k \mid k)$ and the inputs $\hat{\beta}(k-1 \mid k)$ given all the available observations (prior and including time $k$ ). The EKF asymptotic convergence for observable systems is proved in [17]. In [4], the optimality conditions for the KF-UI-WDF are analysed. The only restriction of this filter is to impose that the dimension of the outputs has to be larger than that of the inputs $(n+1>L)$, to ensure the uniqueness of the estimated variables. For the extended case, $n+1$ has to be larger than or equal to $M+L$, where $M$ is the length of the parameters vector.

Unfortunately, like the KF, the limitations of this filter are the hypotheses on the model and measurements noises (only additive noises) and the need of a perfect knowledge of the 
covariance matrices $W$ and $V$. Since we are considering a deterministic framework, these matrices are regarded as tuning parameters. Nevertheless, the EKF has proved its performances in practice even with some missing knowledge, provided that all the implementation steps listed in [18] are respected. Since the final objective of this paper is to propose a practical method for the problem of combined diffusivity and source term estimation in tokamak plasmas, the reaction coefficient $\tau$ is assumed to be known and constant, given by the empirical model [1]. This is convenient since the study of the matrix $\varphi$ defined in subsection III-A using available real data shows that the rank remains full but the conditioning number is close to computer's precision if $\tau$ is estimated, which indicates poor identifiability conditions with this term.

\section{Simulation and experimental results}

The reconstruction performance of our method is evaluated using simulations with computed and experimental data.

1) Illustrative example: The mock-up data is generated using:

$\left\{\begin{array}{l}\chi_{e}(z, t)=\left(0.1+5 z+2 z^{2}+4 z^{3}\right) \mathbf{1}(t)\left(m^{2} / s\right) ; \tau=0.1(s) \\ S(z, t)=\frac{10^{5}}{\sqrt{2 \pi \sigma}} \exp \left(\frac{-(z-\mu)^{2}}{2 \sigma^{2}}\right) \mathbf{g}(t)(e V / s) \\ z \in[0,1], \quad t \in[0,26], \quad d z=0.05, \quad d t=0.01 .\end{array}\right.$

The choice of $\chi_{e}, \tau$ and $S$ is motivated by the example proposed in [19] where it is assumed that the diffusion coefficient has a monomial monotonically increasing function and the heating source undergoes a spatial Gaussian form. $\chi_{e}$ is considered constant in time $(\mathbf{1}(t)=1 \forall t \in[0,26])$, whereas the time variations of $S$ are given by the three-step function $g$ defined by:

$$
\mathbf{g}(t)= \begin{cases}1 & \text { if } t \in[0,1 / 3[ \\ 1.5 & \text { if } t \in[1 / 3,2 / 3[ \\ 2 & \text { if } t \in[2 / 3,1[\end{cases}
$$

Fig. 2 shows that using the cubic b-splines basis functions $\left\{\pi_{i}\right\}_{i=0}^{9}$ for both $\chi_{e}$ and $S$, the approximation error of $\chi_{e}$ is of the order of $10^{-15}$ and the relative approximation error of $S$ is around $10^{-6}$. This led us to take $n \geq 20$ (see IIIA) for the temperature basis. To evaluate the reconstruction
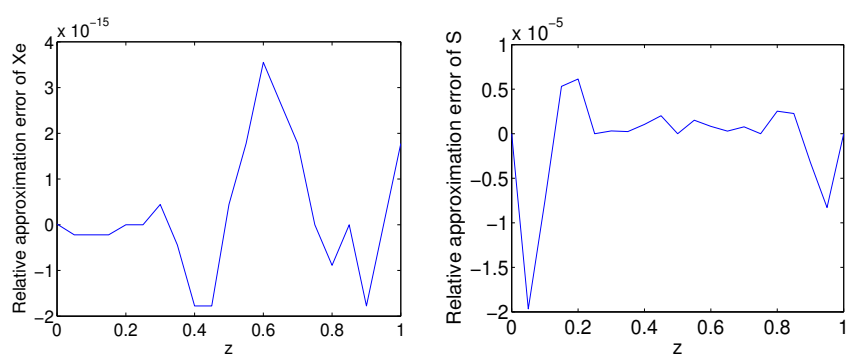

Fig. 2: Approximation errors of $\chi_{e}$ and $S$ for the mock-up example

performance using the EKF-UI-WDF, the initialization of the filter was arbitrary. The covariance matrix of the model noise $W$ is taken equal to $10^{-1} I_{n+M+1}$, the measurements covariance noise $V$ is set to $10^{-3} I_{n+1}$ and the initial state estimation error covariance matrix is $10^{3} I_{n+M+1}$. From
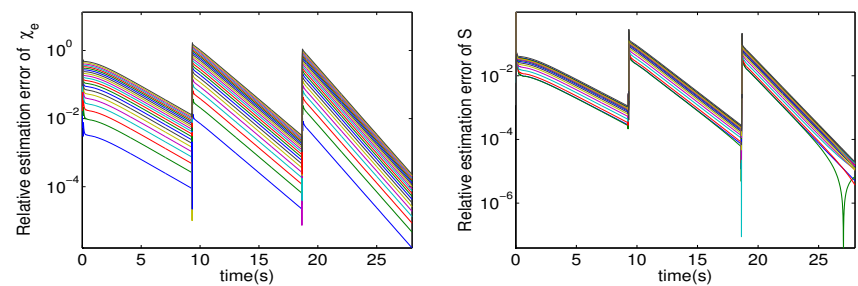

Fig. 3: Estimation errors of $\chi_{e}$ and $S$ for the mock-up example

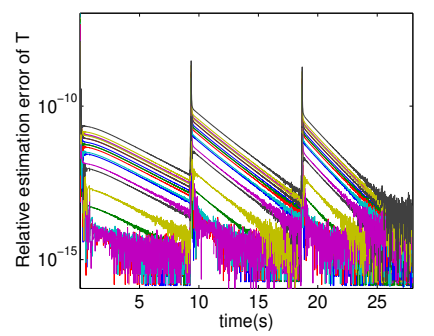

Fig. 4: Relative estimation error of the temperature profile $T$ for the mock-up example

figures 3 and 4, the estimation of $\chi_{e}, S$ and $T$ using the EKFUI-WDF is very good. The filter needs only few iterations to converge to the original variables despite the abrupt changes in the source term $S$.

2) Experimental results: Our estimation strategy is implemented on data provided by the Tore Supra tokamak. It is a large tokamak with a superconducting toroidal magnet, and with a plasma minor radius, $a=0.72 \mathrm{~m}$ and major radius $R=2.4 \mathrm{~m}$. We consider the discharge TS 33632, where the heating is mainly due to the radio-frequency power at the Ion Cyclotron Resonant Heating (ICRH) and the measured temperature and input power $P_{e_{t o t}}$ are provided using diagnostic systems. As in section II, we are dealing with the joint estimation of the diffusion coefficient $\chi_{e}$ and the source term $S . \tau$ is fixed using the empirical law given in [1]. For the projection method, the temperature projection basis, is $n=21$ (since the $\Omega$ is divided into 21 uniform meshes). For $\chi_{e}$ and $S$, the orders of the bases are $M=L=10$. The numerical values for $d z, d t, V$ and $W$ are chosen as in the previous section. Fig. 5 presents the estimated profiles of $\chi_{e}$ and $S$ in the spatial validity interval $(z \leq 0.8)$. Both are positive without enforcing this constraint in the Kalman filter criterion. The EKF-UI-WDF performs well since the relative estimation error of $T$ in Fig. 6 is around $10^{-2 \%}$, the trace of $P_{x}(k \mid k)$ is decreasing and the standard deviation converges to $0.5 \mathrm{eV}$. The superposition of the input power $\left(P_{e_{t o t}}\right.$, in red dashed-line $)$ and the estimated absorbed power (Estimated $P_{e}$, continuous blue line) is presented in Fig.7. The temporal power form is coherent with 

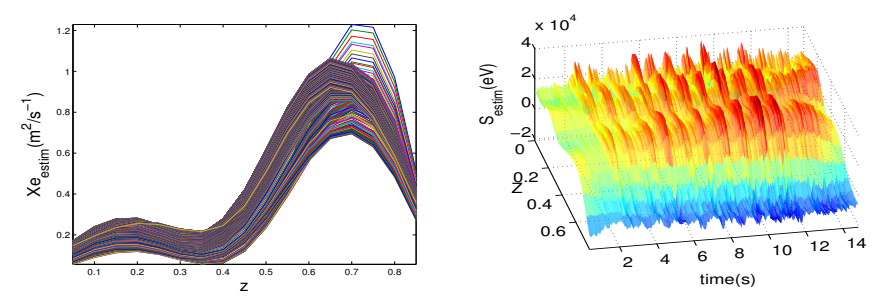

Fig. 5: Estimated profiles of $\chi_{e}$ and $S$
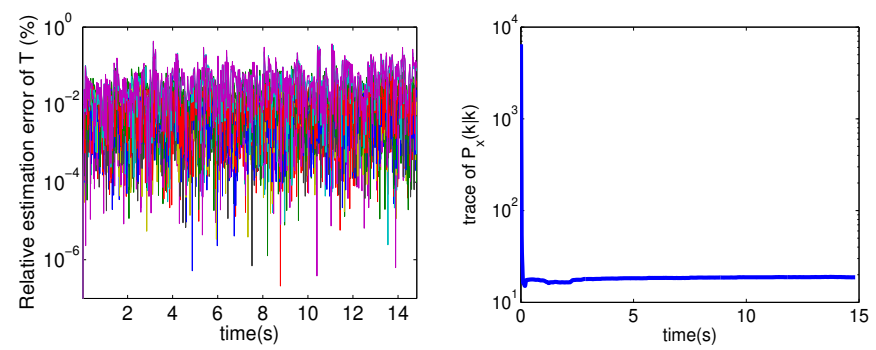

Fig. 6: Relative estimation error of $T$ and the trace of $P_{x}(k \mid k)$

the estimated profile, with a different magnitude probably due to energy losses. The estimated $\chi_{e}$ close to $1\left(\mathrm{~m}^{2} / \mathrm{s}\right)$ is consistent with the expected physical value.

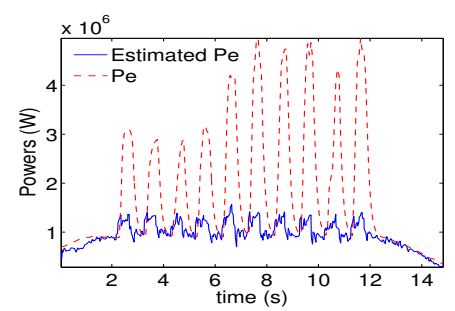

Fig. 7: $P_{e_{t o t}}$ and Estimated $P_{e}$

\section{CONCLUSION}

In this paper we discussed a method for estimating distributed parameters in a finite dimensional framework for linear parabolic PDEs. First we dealt with the space discretization problem, where a cubic b-splines finite element method was chosen. This widely used method provides accurate approximate solutions with sufficient smoothness for direct problems. In the second part, using Galerkin formulation, the spatio-temporal problem was reduced to a state-space time-varying parameter model, and then the EKFUI-WDF was used to estimate simultaneously the states, the parameters and the inputs. Simulation and experimental results testified the interest of the adopted methodology. To overtake the EKF restrictions, other filters like the UKF, $H_{\infty}$ or particle filter can be used, combined with the squareroot implementation, to guarantee the stability and to fix the conditioning number problem. This strategy will be the subject of future studies.

\section{ACKNOWLEDGEMENT}

This work was carried out within the framework of the European Fusion Development Agreement and the French Research Federation for Fusion Studies. It is supported by the European Communities under the contract of Association between EURATOM and CEA. The views and opinions expressed herein do not necessarily reflect those of the European Commission. This project receives partial funding provided by the Bonus Quality Research of Grenoble-INP.

\section{REFERENCES}

[1] G. Hoang, B. Saoutic, L. Guiziou, V. Basiuk, A. Bécoulet, R. Budny, F. Clairet, L. Colas, P. Devynck, C. Gil, E. Joffrin, X. Litaudon, J.L. Ségui, I. Voitsekhovitch, and X. Zou, "Magnetic shear effects on confinement and electron heat transport in Tore Supra discharges with electron heating," Nuclear Fusion, vol. 38, no. 1, pp. 117-132, 1998.

[2] H. Banks and P. Lamm, "Estimation of variable coefficients in parabolic distributed systems," IEEE Transaction on Automatic Control, vol. 4, pp. 386-389, 1985.

[3] C. de Boor, A practical guide to splines, N. Y. Springer, Ed. Applied mathematics series, 1978, vol. 27.

[4] S. Pan, H. Su, H. Wang, J. Chu, and R. Lu, "Input and state estimation for linear systems: A least squares estimation approach," in Proceedings of the 7th Asian Control Conference, Hong Kong, China, August 27-29 2009, pp. 378-383.

[5] G. Bellu, M. P. Saccomani, S. Audoly, and L. D'Angiò, "Daisys: A new numerical software tool to test global identifiability of biological and physiological systems," Comput. Meth. Programs Biomed., vol. 88, pp. 52-61, 2007.

[6] F. Hinton and R. Hazeltine, "Theory of plasma transport in toroidal confinement systems," Reviews of Modern Physics, vol. 48, no. 2, pp. 239-308, 1976.

[7] L. C. Evans, Partial differential equations: Graduate studies in Mathematics, 2nd ed. American Mathematical Society, 2010, vol. 19.

[8] P. M. Prenter, Splines and variational methods, Pure and applied mathematics, Eds. Jhon Wiley \& Sons, 1989.

[9] S. Mechhoud, E. Witrant, L. Dugard, and D. Moreau, "Joint diffusivity and source estimation in tokamak plasma heat transport," American Control Conference, June 2013.

[10] W. Yu and J. H. Seinfeld, "Identification of parabolic distributed parameter systems by regularization with differential operators," Journal of mathematical analysis and applications, vol. 132, pp. 365-387, 1988.

[11] A. A Oberai, N. H. Gokhale, and G. R. Feijóo, "Solution of inverse problems in elasticity imaging using the adjoint method," Inverse Problems, vol. 19, pp. 297-313, 2003.

[12] J. Jacquez and P. Greif, "Numerical parameter identifiability and estimability: integrating identifiability, and optimal sampling design," Math. Biosci, vol. 77, no. 1, pp. 201-228, 1985.

[13] A. Raue, C. Kreutz, U. Klingmuller, and J. Timmer, "Addressing parameter identifiability by model-based experimentation," IET. Syst. Biol., vol. 5, no. 2, pp. 120-130, 2010.

[14] N. Meshkat, M. Eisenberg, and J. J. Distefano, "An algorithm for finding globally identifiable parameter combinations of nonlinear ode models using Gröber bases," Math. Biosci, pp. 61-72, 2009.

[15] S. Gillijns and B. D. Moor, "Unbiased minimum-variance estimation for systems with unknown inputs," Automatica, vol. 43, pp. 934-937, 2007.

[16] H. Palanthandalam-Madapusi and D. S. Bernstein, "Unbiased minimum-variance filtering for input reconstruction," in Proceedings of the 2007 American Control Conference, New York city, USA, July 2007, pp. 11-13.

[17] L. Ljung, "Asymptotic behaviour of extended Kalman filter as a parameter estimator for linear systems," IEEE Trans. on Auto. Control, vol. AC-24, no. 1, pp. 36-50, 1979.

[18] D. Simon, Optimal state estimation: Kalman, $H_{\infty}$ and nonlinear approaches. Jhon Wiley \& Sons, 2006.

[19] X. L. Zou, G. Giruzzi, J. F. Artaud, F. Bouquey, A. Clémençon, C. Darbos, R. J. Dumont, C. Guivarch, M. Lennholm, R. Magne, and J. L. Ségui, "Electron heat transport and ECRH modulation experiments in Tore Supra tokamak," Nuclear Fusion, vol. 43, pp. 1411-1420, 2003. 